Revue internationale P.M.E.

Économie et gestion de la petite et moyenne entreprise

Revue

internationale

PME

\title{
Une nouvelle mesure de la stratégie de diversification des PME
}

\section{Paillette Robic}

Volume 6, numéro 3-4, 1994

URI : https://id.erudit.org/iderudit/1008230ar

DOI : https://doi.org/10.7202/1008230ar

Aller au sommaire du numéro

Éditeur(s)

Presses de l’Université du Québec

ISSN

0776-5436 (imprimé)

1918-9699 (numérique)

Découvrir la revue

Citer cet article

Robic, P. (1994). Une nouvelle mesure de la stratégie de diversification des PME. Revue internationale P.M.E., 6(3-4), 9-36. https://doi.org/10.7202/1008230ar

\section{Résumé de l'article}

L'ensemble des outils d'analyse stratégique demeurent trop souvent inadaptés aux PME. Tel est le cas de la mesure de la diversification, plus précisément de sa nature et ses actions de développement. L'article propose une définition et une mesure de la nature de la stratégie de diversification des PME et de son développement comme outil d'aide à l'analyse du comportement stratégique des PME. L'application du modèle auprès d'un échantillon de 22 PME du secteur laitier de l'Ouest de la France montre l'intérêt d'une méthodologie spécifique aux PME. 


\title{
Une nouvelle mesure de la stratégie de diversification des PME
}

\author{
Paulette ROBIC* \\ Université de Nantes
}

\begin{abstract}
RÉSUMÉ
L'ensemble des outils d'analyse stratégique demeurent trop souvent inadaptés aux PME. Tel est le cas de la mesure de la diversification, plus précisément de sa nature et ses actions de développement. L'article propose une définition et une mesure de la nature de la stratégie de diversification des PME et de son développement comme outil d'aide à l'analyse du comportement stratégique des PME. L'application du modèle auprès d'un échantillon de 22 PME du secteur laitier de l'Ouest de la France montre l'intérêt d'une méthodologie spécifique aux PME.
\end{abstract}

\section{ABSTRACT}

The whole of strategic analysis instruments too often remain inadapted for small and medium-sized enterprises (SMEs). This is the case for measuring diversification, or more precisely speaking, his nature and his development actions. This contribution suggests a definition and a measurement of the diversification nature of SMEs and of development as an instrument to help to analyse strategic behaviour in SMEs. The application of the model with sample of twenty-two firms in the dairy industry in the West of France shows the interest of a specific methodology to SMEs.

\section{RESUMEN}

El conjunto los instrumentos de analisis estrategicos quedan demasiado menudo inadaptados a PyME. Es el caso de la medida de la diversificacion, mas precisamente de su naturaleza y de sus acciones de desarrollo. El articulo propone una definicion y una medida de la natura de la estrategia de diversificacion de las PyME y de su desarrollo como instrumento de ayuda al analisis del comportamiento estrategico de las PyME. La aplicacion del modelo

* Paulette Robic est docteur en sciences de gestion, maître de conférences en sciences de gestion à l'Université de Nantes et membre du groupe de recherche C3E «Centre d'étude sur l'économie internationale et l'entreprise » à la Faculté de sciences économiques et de gestion de Nantes. Elle travaille actuellement sur la compétitivité et la politique de prix des PME. Adresse : Faculté de sciences économiques et de gestion, 110, bd Michelet, 44071 Nantes, Cedex 03, France. 
a una muestra de 22 PyME del sector lachero del Oeste de Francia ensena el interès de una metodologia especifica para las PyME.

\section{Introduction}

Chacun, chercheur, gestionnaire d'entreprise ou responsable politique, a pris conscience du rôle joué aujourd'hui par les PME dans le développement économique. Il suffit de consulter les études de plus en plus nombreuses qui leur sont consacrées. Il n'en demeure pas moins que leur comportement stratégique reste difficile à cerner. La rareté des outils d'analyse stratégique adaptés aux PME ne fait que renforcer cette situation.

Ainsi en est-il de la nature de la diversification des PME et de son développement dont le rôle fondamental pour garantir une gestion équilibrée des ressources dans un contexte donné est aujourd'hui reconnu. Proposer une nouvelle mesure de la nature de la stratégie de diversification et de son développement qui soit adaptée aux caractéristiques de la PME comme outil d'analyse de leur comportement stratégique est donc l'objectif recherché ici. Cette proposition se fonde sur une définition de la notion d'activité des PME appropriée aux spécificités de la PME. Elle se fonde également sur l'hypothèse que les PME sont essentiellement présentes dans une seule industrie (Industrie agro-alimentaire, métallurgie...), voire le plus souvent dans un seul secteur.

En référence à cette hypothèse, l'étude se fait selon une approche sectorielle et s'appuie sur l'exemple de 22 PME du secteur laitier de l'Ouest de la France. L'échantillon a été constitué avec les contraintes suivantes. L'entreprise devait:

- avoir entre 10 et 500 salariés ;

- être indépendante de toute entreprise financièrement ;

- avoir pour activité principale la transformation du lait.

Aussi arbitraire soit-il, le critère «effectifs» a été choisi pour définir ce qu'est une PME. Le seuil de 500 salariés, plutôt que 200, a été retenu afin de répondre à la réalité du secteur laitier en France où la concentration est telle que les entreprises de moins de 100 salariés sont de plus en plus rares. Parallèlement, la collecte du lait qui requiert une main-d'œuvre importante contribue également à la diminution des entreprises de moins de 100 salariés dans ce secteur.

Le chiffre d'affaires est parfois préféré aux effectifs pour définir la PME, mais il est aussi arbitraire que les effectifs, notamment, dans le sens où une entreprise avec un faible chiffre d'affaires peut être leader de son marché tandis qu'une autre avec un chiffre d'affaires apparemment élevé ne peut n'avoir que 
peu d'importance sur son marché. Par ailleurs, le chiffre d'affaires est soumis aux fluctuations monétaires, ce qui lui retire tout caractère universel contrairement aux effectifs.

Pour pallier cette lacune, un second critère a été choisi : être indépendante de toute entreprise financièrement. Tant qu'une entreprise de cette taille demeure indépendante financièrement, elle conserve une certaine flexibilité, ses structures ne sont pas trop lourdes et, surtout, son dirigeant en garde le contrôle, y exerce une forte influence. Ce sont là les caractéristiques essentielles de la PME.

En plus de répondre à ces critères de base, les 22 entreprises étudiées ont les caractéristiques suivantes:

TABLEAU 1

Répartition des 22 entreprises selon leurs effectifs

\begin{tabular}{ccc}
\hline Effectifs & $\mathbf{n}$ & \% \\
\hline $10-19$ & 3 & 13,7 \\
$20-49$ & 2 & 9,0 \\
$50-99$ & 6 & 27,3 \\
$100-499$ & 11 & 50,0 \\
\hline Total & $\mathbf{2 2}$ & $\mathbf{1 0 0 , 0}$ \\
\hline
\end{tabular}

TABLEAU 2

Répartition des 22 entreprises selon leur statut juridique

\begin{tabular}{ccc}
\hline Statut juridique & $\mathbf{n}$ & $\%$ \\
\hline SA & 10 & 45,5 \\
SARL & 3 & 13,6 \\
SNC & 1 & 4,5 \\
Coop & 8 & 36,4 \\
\hline Total & $\mathbf{2 2}$ & $\mathbf{1 0 0 , 0}$ \\
\hline
\end{tabular}

Rappelons que ces entreprises sont toutes indépendantes financièrement.

À partir de cet échantillon, un modèle de mesure de la stratégie de diversification des PME et de son développement sera élaboré.

À l'aide de l'analyse typologique qu'est la classification ascendante hiérarchique, le profil de la stratégie de diversification suivie par les 22 PME 
qui constituent l'échantillon sera tracé dans une dernière phase. Cela permettra d'appliquer les propositions avancées au préalable, afférentes à la mesure de la stratégie de diversification des PME.

\section{Comment mesurer la stratégie de diversification des PME ?}

Non seulement la diversification est ici prise sous l'angle statique, mais aussi dynamique. Aussi doit-elle être mesurée selon ces deux vues distinctes. Dans un premier temps, la diversification doit être " photographiée » dans sa nature. Dans un deuxième temps, au contraire, elle doit être «filmée » dans son développement.

\subsection{Photographier la nature de la stratégie de diversification des PME}

La diversification n'a pas fait l'objet de recherches théoriques et empiriques suffisantes pour pouvoir parler d'une théorie de la diversification (Ramanujan et Varadarajan, 1989). Il n'y a que des éléments d'une théorie, ce qui explique les nombreuses définitions proposées de la diversification. Malheureusement, celles-ci se limitent aux problèmes des grandes entreprises et ne s'intéressent pas aux spécificités des PME. Il s'agit donc ici de définir la diversification des PME en tenant compte de leurs caractéristiques avant de proposer une mesure.

\subsubsection{Approche conceptuelle de la diversification}

Bien que différentes et adaptées uniquement à la grande entreprise, les définitions de la diversification proposées jusqu'ici mettent en exergue que la diversification décrit la diversité des activités.

Avant de définir la stratégie de diversification, il faut donc s'interroger sur la notion d'activité des PME; en d'autres termes, réfléchir à la segmentation stratégique de l'activité des PME.

\section{Notion d'activité}

La notion d'activité des PME sera définie comme le choix des technologies (ou produits en tant que concrétisation d'une technologie), des besoins (ou fonctions), des clients et de la zone géographique (France, étranger). Aux produits correspondent les technologies, aux marchés correspondent les besoins, les clients et la zone géographique. 
L'activité des PME ainsi décrite rejoint la proposition d'Abell (1980), puisqu'il la définit en ces termes: «choix des technologies, des fonctions et des clients à servir, non pas des produits à offrir». Elle s'en écarte simplement du fait de la prise en compte simultanée des groupes de clients servis et de la zone géographique desservie au sein de la variable nature des clients.

Explicitement, l'activité des PME du secteur laitier sera considérée comme: le choix des technologies (du yaourt, du lait, du fromage, du jus de fruit...), des fonctions (alimentation humaine et animale...), des clients (collectivités, consommateurs finaux...) et de la zone géographique (France, étranger) à servir, et non pas des produits à offrir.

Le choix des indicateurs des quatre variables qui définissent la notion d'activité des PME, du secteur concerné, tient compte des caractéristiques de ce dernier. Par exemple, l'âge, la catégorie socioprofessionnelle (csp) conviennent à des marchés de consommation strictement finale. Ils deviennent inadéquats lorsque les clients peuvent être également des industriels comme dans le cas du secteur laitier.

\section{Notion de diversification}

Pour proposer, à partir du concept d'activité retenu, une définition de la notion de diversification adaptée aux spécificités des PME, il faut s'aider de celles existantes. Même si elles concernent davantage les grandes entreprises, ces définitions sont des repères indispensables.

Pour Penrose (1963), «une entreprise diversifie ses activités productives chaque fois que sans abandonner entièrement ses anciennes lignes de produits, elle s'engage dans la fabrication de produits nouveaux, y compris de produits intermédiaires suffisamment différents de ses autres fabrications pour entraîner des différences significatives dans les programmes de production ou de distribution de l'entreprise». En concevant la diversification ainsi, Penrose amorce un courant de pensée qui, d'une part, intègre à la fois les aspects techniques et commerciaux et, d'autre part, reconnaît les interdépendances entre les produits ou groupes de produits. En cela, elle s'écarte de ceux qui estiment, comme Thorp (1924), que la diversification implique l'indépendance totale des fonctions de production. Dans cette acception, la seule diversification possible serait le conglomérat.

Avec les travaux de Rumelt (1974), qui distinguent deux types de liaison - linked et constrained -, les travaux de Bienaymé (1971), - diversification mineure et majeure - et de Salter et Weinhold (1979) - diversification liée complémentaire et diversification liée supplémentaire - illustrent les propositions qui ont été faites, pour montrer que plus qu'un nombre de produits et de marchés, la diversification est une diversité de produits, autrement dit, un 
niveau de liaison entre les produits. Pour reprendre les termes de Bienaymé (1971 : 72-73), «la diversification mineure exploite systématiquement l'ensemble des complémentarités interproduits et interfonctions sans accroissement marqué des coûts».

Bien que définissant la diversification de façon étroite comme une «rupture à la fois avec les produits et avec les marchés familiers à l'entreprise», Ansoff (1965: 94) s'inscrit dans cette même veine de pensée. Les quatre formes de diversification qu'il distingue - diversification horizontale, intégration verticale, diversification concentrique et diversification hétérogène (conglomérale)sont établies selon le lien entre les nouveaux produits et les produits existants, ainsi qu'en témoignent les missions nouvelles et les marchés existants.

Il ressort de ces différentes acceptions de la diversification qu'une entreprise qui n'a qu'un seul produit est spécialisée selon ce critère. Une entreprise a un degré de diversification d'autant plus élevé que le nombre de ses produits est élevé. Parallèlement, une entreprise aura un degré de diversification par rapport aux marchés d'autant plus fort que leur nombre sera élevé également. Une entreprise peut donc être diversifiée par rapport aux produits et spécialisée par rapport aux marchés, ou l'inverse, et être aussi diversifiée selon ces deux critères. Conjointement au nombre d'activités, la diversité des produits est un critère de définition de la diversification. Une entreprise peut être diversifiée selon ses produits, mais spécialisée selon les ressources si les liaisons sont fortes. L'utilisation d'une même ressource pour plusieurs produits peut faire l'objet d'une spécialisation. Chaque critère peut être la base d'une diversification ou spécialisation (Bamberger, 1981).

Dans ces différentes propositions de définition de la diversification, chacun parle de produit sans en déterminer le sens ni le niveau d'abstraction. Correspond-il à une technologie ? Est-il pris dans le sens physique, dans le sens groupe de produits ou comme une branche? Il est bien entendu que nous le considérons ici comme la résultante d'une technologie. Par ailleurs, l'étude se situant, d'une part, au niveau des PME et, d'autre part, au niveau d'un seul secteur, la notion de produit ne peut être ramenée à la notion de branche. Autrement, l'analyse du comportement de diversification de ces entreprises risquerait d'aboutir à l'étude du comportement de spécialisation.

Le niveau d'abstraction de la notion de produit le mieux adapté à l'étude présente paraît être le groupe de produits, en référence à l'idée qu'une technologie est non pas la source d'un seul produit, mais d'un groupe de produits.

Le produit défini en ces termes implique, par exemple, la non-différenciation des laits. Le produit lait comprend l'ensemble des laits (UHT, stérilisé, etc.). 
La définition de la diversification des PME doit tenir compte de deux réalités essentielles concernant les entreprises de cette taille:

- la liaison entre les produits est forte et quasi générale ;

- leurs marchés sont rarement totalement hétérogènes.

Conformément à la définition de la notion d'activité, à ces caractéristiques des PME et à partir des définitions existantes, la diversification des PME se traduira par le nombre, l'importance et la diversité de produits, d'une part, et correspondra au nombre de groupes de clients servis, de besoins satisfaits, ainsi qu'à l'étendue des marchés de l'entreprise au niveau des groupes de clients servis, des groupes de besoins satisfaits et de la zone géographique desservie, d'autre part.

La diversification ainsi définie tient compte de la forte interdépendance entre les produits puisqu'elle intègre simultanément le nombre de produits et leur importance dans le sens ventilation du chiffre d'affaires, mais aussi leur diversité dans le sens degré de liaison entre les produits. Elle tient également compte de l'homogénéité des marchés puisqu'elle ne considère pas le marché comme une entité à part entière, mais comme une combinaison de groupes de clients, de groupes de besoins et de zones géographiques définies plus ou moins étroitement par l'entreprise. De cette façon, le niveau et la forme de la diversification sont simultanément mesurés.

La diversification des PME quant à leurs produits sera donc une combinaison de leur nombre, de leur importance (ventilation du chiffre d'affaires) et de leur diversité (liaison entre les produits).

De la même façon, la diversification des PME, donc celles du secteur laitier, par rapport à leurs marchés est définie en raisonnant au niveau des trois dimensions qui ont été choisies précédemment pour déterminer les marchés de ces entreprises dans le cadre de la définition de la notion d'activité, à savoir:

- le groupe de clients servis ;

- le groupe de besoins satisfaits ;

- la zone géographique desservie.

À partir de ces trois variables, par exemple, la diversification des PME du secteur laitier par rapport à leurs marchés consistera, d'une part, en un certain nombre de groupes de clients servis (collectivités, consommateurs finaux...), un certain nombre de groupes de besoins satisfaits (alimentation humaine, alimentation animale...) et, d'autre part, en une certaine étendue des différents groupes de clients servis (p. ex.: consommateur $\leq 20$ ans ou $\geq 20$ ans), des groupes de besoins satisfaits (p. ex.: goûter, boisson...) et de la zone géographique desservie (nationale ou internationale). 
La variable «zone géographique» permet de scinder les marchés des entreprises en marchés nationaux et internationaux, voire en activité nationale et internationale, en intégrant les produits aux groupes de clients et aux besoins satisfaits.

Une entreprise peut être diversifiée selon un ou plusieurs des trois critères. Elle peut servir plusieurs groupes de clients et satisfaire un groupe de besoins spécifiques définis plus ou moins largement, sur le plan national ou international. Une autre entreprise peut répondre à plusieurs groupes de besoins, définis largement, sur le plan national, pour un seul groupe de clients défini étroitement. Ainsi, une entreprise peut être spécialisée selon les groupes de besoins satisfaits et les groupes de clients servis, mais être diversifiée géographiquement dans le sens où elle couvre les marchés internationaux. La figure 1 permet de visualiser certaines options qui s'offrent aux PME laitières quant aux groupes de clients qu'elles servent et aux besoins qu'elles satisfont, que ce soit au niveau national ou international.

FIGURE 1

Exemple de possibilités de diversification par rapport aux groupes de clients et aux besoins satisfaits

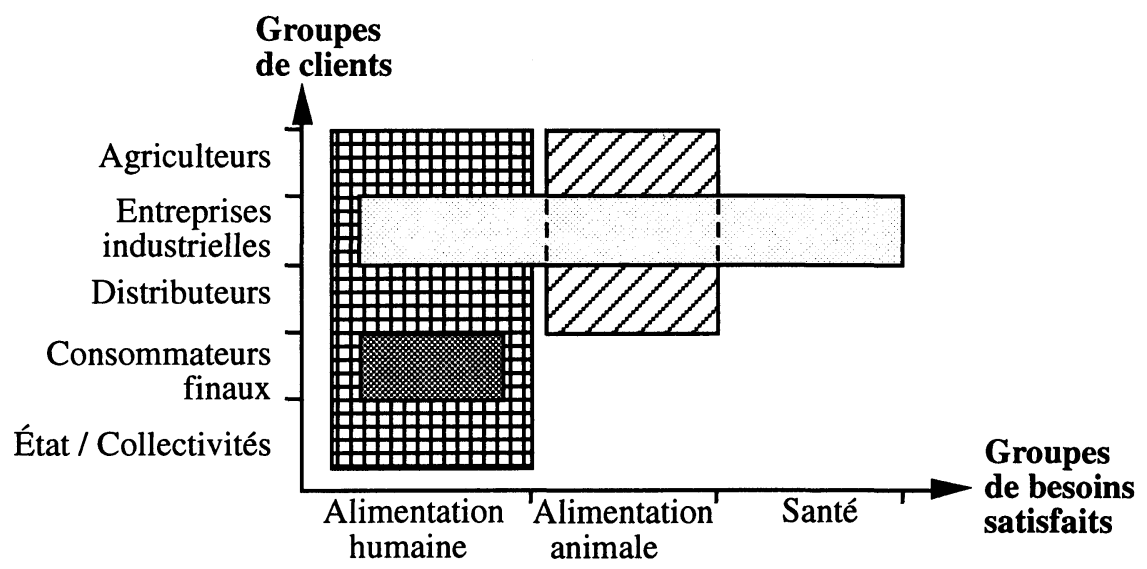

L'étendue du marché est le résultat de la conjonction de ces trois critères. Dans le secteur laitier, définir son marché comme la satisfaction du besoin alimentaire qu'est le goûter des consommateurs âgés de moins de 20 ans en France est un exemple des extrêmes possibles de l'étendue. À l'opposé, il est envisageable de définir son marché comme la satisfaction des besoins de goûter, dessert, boisson... pour les consommateurs de toutes les tranches d'âge en France et à l'étranger. 
La combinaison des produits et des variables qui définissent les marchés des PME peut se traduire, par exemple, par une spécialisation par rapport aux produits et aux groupes de clients satisfaits et une diversification par rapport aux groupes de besoins satisfaits, avec une intervention à l'échelle nationale et internationale. De nombreux cas de figure existent, chaque dimension pouvant être l'objet d'une diversification ou d'une spécialisation. La figure 2 en représente certains pour le cas des PME du secteur laitier.

\section{FIGURE 2}

\section{Exemple d'alternative de diversification des activités d'une PME laitière qui exporte}
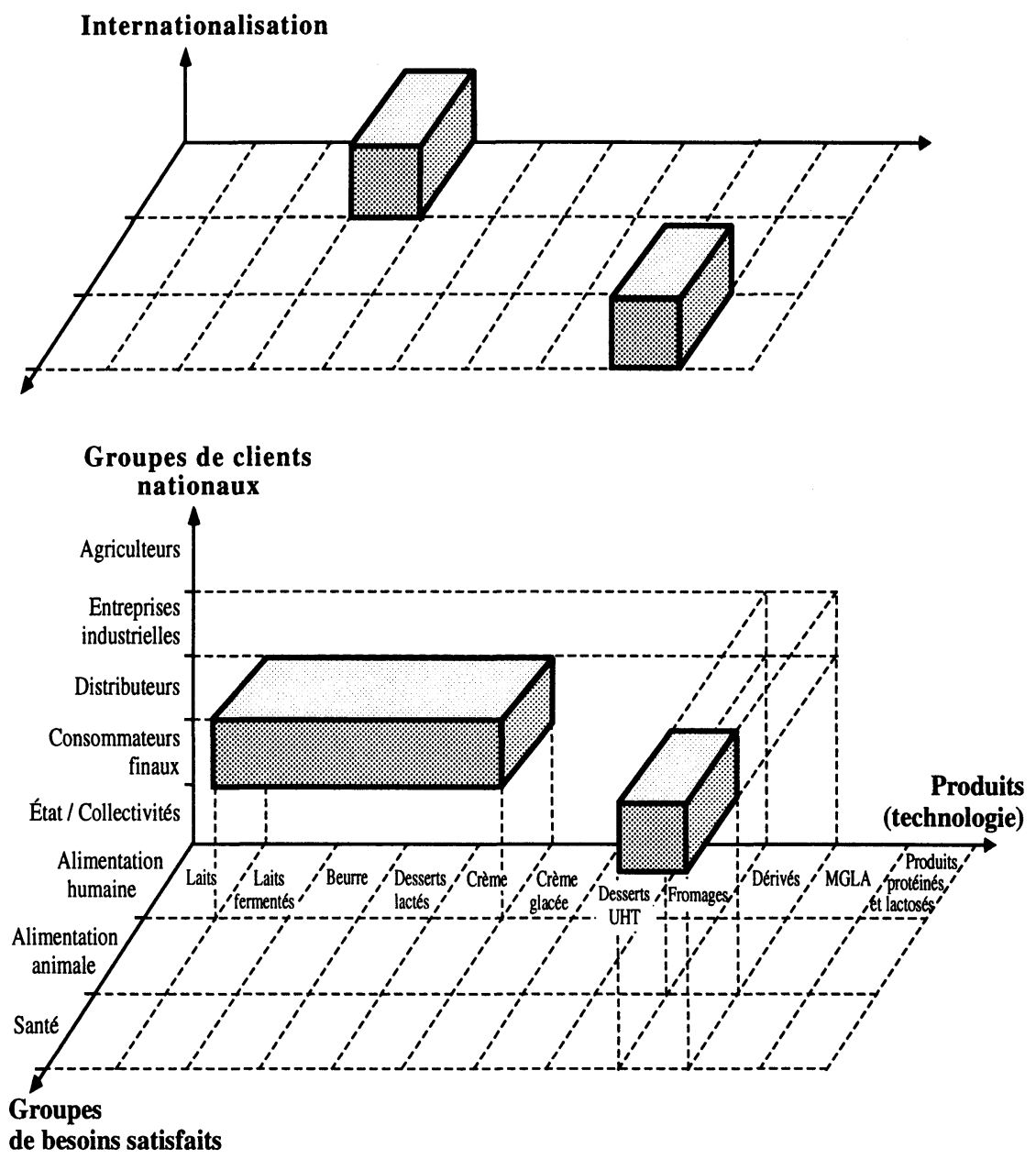

Revue internationale P.M.E., vol. 6, nos 3-4, 1994 
Contrairement aux définitions proposées par la théorie dont les principales ont été présentées ici, cette définition permet de considérer l'activité stratégique de l'entreprise dans ses détails, et non pas dans sa globalité, comme le font les approches qui s'intéressent aux grandes entreprises.

La diversification des PME définie, il convient, à partir du cas des PME du secteur laitier, de proposer une mesure du niveau et de la forme de la diversification des PME. En d'autres termes, il faut déterminer un ou des indicateurs de la diversification.

\subsubsection{Mesure du contenu de la diversification}

Les propositions afférentes à la mesure de la diversification qui existent actuellement peuvent être regroupées en deux catégories (Robic, 1989). Les premières s'inspirent des travaux de l'économie industrielle et sont de type quantitatif. Elles cherchent à comparer le niveau de diversification entre les entreprises. Les secondes, dont la principale est celle de Rumelt (1974), visent à cerner la logique qui anime les entreprises dans leur stratégie de diversification. Comparées aux autres propositions, celles-ci relèvent de la micro-économie et sont dites catégorielles.

Les propositions quantitatives ont trois qualités: la fiabilité, la simplicité et l'économie de temps. En revanche, elles ont pour effet de réduire la mesure de la diversification des activités des entreprises à la mesure de la diversification des produits en ignorant, qui plus est, les liaisons entre les produits. La proposition de Rumelt a une certaine validité pour la grande entreprise. Par contre, elle est difficilement applicable à la PME, car elle repose sur une définition de l'activité où les liens entre activités sont très faibles, voire nuls, ce qui ne correspond guère à la réalité des PME.

Compte tenu des limites de ces mesures, celle que nous proposons ici s'écarte totalement de la mesure de Rumelt; elle s'inspire des mesures quantitatives en les adaptant aux caractéristiques des PME:

- forte interdépendance entre les groupes de produits ;

- homogénéité des marchés relativement forte.

Le souci de l'étude est de mesurer le niveau et la forme de la diversification des PME, d'une part, selon chacune des dimensions qui la définissent et de façon globale, d'autre part.

Comme un seul indicateur ne peut permettre cette approche détaillée puis globale, nous en proposerons plusieurs. L'objectif est de les rendre additifs et comparables, pour cela, il faut qu'ils aient le même poids. L'amplitude [0 - 1] sera choisie comme base de référence de cette propriété d'additivité et de comparabilité. 
La diversification des produits sera mesurée par DP

où : $\quad \mathrm{DP}=\frac{\mathrm{DI}+\mathrm{DS}+\mathrm{DV}}{3}$

avec DI indicateur de la diversification intersectorielle

DS indicateur de la diversification sectorielle

DV indicateur de la diversité des produits (liaisons entre les produits).

DI, indicateur de la diversification intersectorielle des PME, s'écrit :

$$
\mathrm{DI}=\frac{1}{\log a}\left(\sum_{j=1}^{k} z_{j} \cdot \log \frac{1}{z_{j}}\right)
$$

où : $\quad z_{j}$ est le rapport entre le chiffre d'affaires de l'entreprise réalisé dans le secteur $j$ (p. ex.: laitier) et son chiffre d'affaires total réalisé dans les secteurs $k$ (p. ex.: viande, lait, conserves)

et : $\quad \log \frac{1}{z_{j}}$ secteur $j$.

permet de pondérer chaque activité réalisée dans chaque

Cela donne un poids proportionnellement moins élevé aux activités les plus importantes.

$\log a$ est la diversification intersectorielle maximale que peut réaliser une PME ( $a=7$ pour les PME du secteur laitier en regard de la nomenclature à 2 chiffres proposée par l'INSEE dans la mesure où il est plausible de considérer qu'elles ne sortent pas de l'IAA).

$\frac{1}{\log a}$

permet à DI d'avoir une amplitude comprise entre 0 et 1 . Cela lui donne une propriété d'additivité et de comparabilité vis-à-vis des indicateurs des autres dimensions, DS et DV.

DI, qui s'inspire de l'indicateur d'entropie (Jacquemin et Kumps, 1971), permet de voir si les PME étudiées ont des activités dans des secteurs en dehors de leur secteur d'activité principale (p. ex.: le secteur laitier). 
DS, indicateur de la diversification sectorielle des PME, s'écrit:

$$
\mathrm{DS}=\frac{1}{\log b}\left(\sum_{i=1}^{n} z_{i} \cdot \log \frac{1}{z_{i}}\right)
$$

où : $\quad z_{i}$ est la part du chiffre d'affaires de l'entreprise réalisée dans le soussecteur d'activité principale $i$ (p. ex. : lait , fromage pour le secteur laitier) par rapport au chiffre d'affaires total de l'entreprise réalisé dans les $n$ sous-secteurs du secteur d'activité principale où elle est présente

et : $\quad \log b$ est la diversification sectorielle maximale que peut réaliser une PME dans son secteur d'activité principale ( $b=11$ dans le secteur laitier).

\section{$\frac{1}{\log b}$} 0 et 1.

permet comme pour DI d'avoir une amplitude comprise entre

DS, qui s'aide également de l'indicateur d'entropie, mesure la diversification dans le seul secteur d'activité principale (p. ex. : le secteur laitier), autrement dit, la diversification sectorielle comme, par exemple, la diversification laitière.

Les PME étant rarement présentes de façon intensive dans deux secteurs d'activité, il semble opportun de limiter l'analyse de la diversification sectorielle au seul secteur d'activité principale.

La nomenclature à 6 chiffres de l'INSEE est utilisée pour calculer la diversification sectorielle (laitière). Dans le cas des PME du secteur laitier, le nombre $b$ de sous-secteurs où celles-ci peuvent être actives est, par exemple, de 11 (fromage, lait, lait fermenté, dérivés).

L'indicateur d'entropie, comme les autres mesures quantitatives, s'avère insuffisant comme outil de mesure des liaisons entre les produits.

DV, l'indicateur des liaisons entre les produits, s'écrit:

$$
\mathrm{DV}=1-\frac{\mathrm{I}}{\mathrm{L}}
$$

où : I est le nombre de liaisons existantes

et $\mathrm{L}$ est le nombre de liaisons possibles.

Les facteurs susceptibles d'être une source de liaison entre les produits sont, notamment, les matières premières principales, les matières premières secondaires, les équipements et les compétences professionnelles. 
Le nombre de critères retenus comme sources de liaisons possibles dans le cadre du secteur laitier est égal à 10 , donc $\mathrm{L}=10$ pour la mesure de la diversité des produits des PME du secteur laitier ${ }^{1}$.

La diversification marché sera mesurée en considérant le marché non pas comme une entité, mais en le scindant suivant les critères qui le définissent. Elle s'écrira ainsi :

$$
\mathrm{DM}=\frac{\mathrm{DC}+\mathrm{DB}+\mathrm{DG}}{3}
$$

avec DC indicateur de la diversification clients

DB indicateur de la diversification besoins

DG indicateur de la diversification géographique.

Les dimensions groupes de clients et groupes de besoins ont la même structure; aussi les indicateurs de la diversification clients et besoins peuventils être abordés simultanément.

DC et DB s'écriront:

$$
\begin{aligned}
& \mathrm{DC}=\left[a\left[\left(\frac{x}{\mathrm{X}}+\frac{y}{\mathrm{Y}}\right)\left(\frac{1}{\mathrm{~W}}+\frac{1}{\mathrm{Z}}\right)\right]\right]^{2} \\
& \mathrm{DB}=\left[a\left[\left(\frac{w}{\mathrm{~W}}+\frac{z}{\mathrm{Z}}\right)\left(\frac{1}{\mathrm{X}}+\frac{1}{\mathrm{Y}}\right)\right]\right]^{2}
\end{aligned}
$$

où : $\quad x$ est le nombre de groupes de clients réellement servis

$\mathrm{X}$ est le nombre de groupes de clients possibles

$y$ est le nombre de types de groupes de clients réellement servis

$\mathrm{Y}$ est le nombre de types de groupes de clients possibles

avec: les types de groupes de clients servis qui caractérisent l'étendue des groupes de clients servis

et où : $w$ est le nombre de groupes de besoins réellement satisfaits

$\mathrm{W}$ est le nombre de groupes de besoins possibles

$z$ est le nombre de types de groupes de besoins réellement satisfaits

$\mathrm{Z}$ est le nombre de types de groupes de besoins possibles

1. Ces critères sont : les matières premières principales, les matières premières secondaires, les techniques de fabrication fondamentales, les équipements, les compétences professionnelles, les circuits d'approvisionnements, les clients, les circuits de distribution, les besoins identiques de la clientèle, les mêmes comportements d'achat. 
avec : les types de groupes de besoins qui caractérisent l'étendue des groupes de besoins satisfaits

où $\left(\frac{1}{W}+\frac{1}{Z}\right)$

, la proportion minimum de la diversification de groupes et de types de groupes de besoins satisfaits, pondère la proportion de groupes de clients servis par chaque entreprise

et $\left(\frac{1}{X}+\frac{1}{Y}\right)$

, la proportion minimum de groupes et de types de clients servis, pondère la proportion de groupes et types de besoins satisfaits par chaque entreprise.

$$
\mathrm{a}=\frac{1}{\left(\frac{x}{\mathrm{X}}+\frac{y}{\mathrm{Y}}\right)\left(\frac{1}{\mathrm{~W}}+\frac{1}{\mathrm{Z}}\right)} \quad \mathrm{b}=\frac{1}{\left(\frac{w}{\mathrm{~W}}+\frac{z}{\mathrm{Z}}\right)\left(\frac{1}{\mathrm{X}}+\frac{1}{\mathrm{Y}}\right)}
$$

permettent d'obtenir une valeur maximale de DC et DB égale à 1 .

Pour les PME du secteur laitier, par exemple, le nombre de groupes et de types de groupes de clients possibles est respectivement de 5 et 12, et le nombre de groupes et de types de groupes de besoins possibles est respectivement de 3 et 8 (voir supra p. 13).

Ces indicateurs offrent l'intérêt de prendre simultanément en compte le nombre et l'étendue, soit des groupes de clients réellement servis pour DC, soit des groupes de besoins réellement satisfaits pour DB relativement aux groupes de clients qu'il est possible de servir et aux groupes de besoins qu'il est possible de satisfaire pour les PME du secteur concerné.

DG, indicateur de la diversification géographique, s'écrit:

$$
\mathrm{DG}=\frac{\mathrm{Y}}{\mathrm{X}}
$$

où : $y$ est égal au chiffre d'affaires réalisé à l'exportation

et $\quad x$ est égal au chiffre d'affaires total de l'entreprise.

Cet indicateur permet de savoir si l'entreprise a ou non une activité internationale, et d'évaluer sa propension à exporter lorsqu'elle exporte. 
La diversification totale des entreprises s'écrira:

$$
\mathrm{D}=\frac{\mathrm{DP}+\mathrm{DM}}{2}
$$

L'amplitude de ces différents indicateurs est comprise entre [0 - 1]. L'un des intérêts de cette mesure de la diversification est d'être décomposable et donc composable suivant les différentes dimensions qui définissent la diversification. Par conséquent, elle tient compte des caractéristiques des PME. Un autre intérêt de cette mesure est d'être généralisable à toute étude sectorielle de la diversification des PME. Il revient aux chercheurs ou gestionnaires intéressés par celle-ci de :

- déterminer le nombre de secteurs dans lesquels les entreprises peuvent être actives en dehors du secteur étudié ;

- déterminer le nombre de sous-secteurs dans lesquels les entreprises peuvent être actives au sein du secteur étudié ;

- fixer les critères de liaisons entre les produits ;

- déterminer les groupes et types de groupes de clients qu'il est possible de servir dans le secteur ;

- déterminer les groupes et les types de groupes de besoins qu'il est possible de satisfaire dans le secteur concerné.

L'échelle utilisée pour déterminer si une entreprise est plus ou moins diversifiée selon les dimensions considérées une à une et selon la totalité de la diversification, donc selon la valeur de $\mathrm{D}$, est celle-ci :

- si la valeur de l'indicateur est 0 , la diversification n'existe pas ;

- si la valeur de l'indicateur est $[0,001-0,159]$, la diversification est très faible ;

- si la valeur de l'indicateur est [0,160 - 0,259], la diversification est faible ;

- si la valeur de l'indicateur est [0,250-0,509], la diversification est moyenne ;

- si la valeur de l'indicateur est $[0,510-0,759]$, la diversification est forte ;

- si la valeur de l'indicateur est $[0,760-1]$, la diversification est très forte. 
Ainsi une entreprise pour laquelle:

$\mathrm{DI}=0,028 ; \mathrm{DS}=0,411 ; \mathrm{DV}=0,8 ; \mathrm{DP}=0,413 ; \mathrm{DC}=0,765 ; \mathrm{DB}=0,879$; $\mathrm{DG}=0,05 ; \mathrm{DM}=0,564$;

$\mathrm{D}=0,490$ est considérée comme ayant :

- une diversification intersecteurs très faible ;

- une diversification laitière (sectorielle) moyenne ;

- une diversité des produits très forte ;

- une diversification produits moyenne ;

- une diversification clients très forte ;

- une diversification besoins très forte ;

- une diversification géographique très faible ;

- une diversification marchés forte ;

- une diversification globale moyenne.

La nature de la diversification des PME photographiée, il faut mesurer ou filmer les manœuvres stratégiques engagées qui s'y rattachent.

\subsection{Filmer les actions de développement des PME}

Les actions de développement alliées à la nature de la diversification peuvent contribuer efficacement à l'identification du comportement de diversification des PME. Mais avant de les mesurer, il est nécessaire de donner leur définition.

\subsubsection{Notion d'action de développement}

Par action de développement sur les activités produit/marché, il faut entendre les changements opérés sur ces activités, plus exactement, leur orientation et le type de variables qui définissent les notions de produit et marché concernées par ces changements.

Vouloir définir la notion d'action de développement des PME sur leurs activités produit/marché signifie déterminer, dans un premier temps, l'orientation des changements que peuvent effectuer ces entreprises et, dans un second temps, cela signifie relever, parmi les variables qui définissent la notion d'activité produit/marché, celles qui, dans le cadre de ces entreprises, sont susceptibles d'être soumises à des changements tels qu'ils permettent de discriminer ces entreprises. 
À l'examen de la réalité des entreprises et des résultats de différentes études empiriques menées auprès de PME (Chicha et Julien, 1979; CEPME, 1980) avec Rothschild (1979), il ressort que les PME effectuent des changements à l'égard de leurs activités qui peuvent être: le repli, le maintien et l'expansion.

Parmi les critères qui définissent la notion d'activité produit/marché, les produits, plus précisément leur nombre et leur liaison, seront retenus comme facteurs de discrimination des actions de développement des PME relatives à leurs activités stratégiques.

L'étude considère que l'importance des différents produits, dans le sens contribution au chiffre d'affaires, évolue surtout sous l'influence des modifications afférentes au nombre de produits. Aussi, à l'inverse du nombre de produits, l'importance des produits ne sera-t-elle pas admise comme un élément de discrimination des actions de développement des PME sur leurs activités.

Plus encore que les changements opérés par rapport au nombre de produits, ce sont les changements effectués par rapport aux liaisons entre les produits qui doivent être analysés dans le cadre des PME, puisque celles-ci se caractérisent très souvent par une forte liaison entre les groupes de produits. Les liaisons entre les produits seront donc considérées comme une variable discriminante des actions de développement des PME sur leurs activités produit/marché.

Bien que la nature des groupes de clients des PME ne doive pas évoluer aussi rapidement que celle des produits, surtout sur des marchés saturés, celleci peut être considérée comme un élément de la stratégie de développement tel, qu'il faut le prendre en compte pour connaître la réalité des actions de développement des PME. Les travaux de Chicha et Julien vont d'ailleurs dans ce sens. Toutefois, la nature des groupes de clients des PME ne doit pas changer suffisamment pour que les changements à l'égard de l'étendue des groupes de clients soient analysés. Seuls les changements par rapport au nombre de groupes de clients seront examinés chez les PME.

Les groupes de besoins satisfaits, que ce soit leur nombre ou leur étendue, peuvent être admis comme relativement stables dans le cadre des entreprises de cette taille.

Dans une industrie, le nombre de groupes de besoins à satisfaire est plus limité que le nombre de produits offerts. Un groupe de besoins peut être satisfait par plusieurs produits. Si l'hypothèse de changements relativement fréquents a été adoptée quant aux produits, il est plausible d'admettre que les PME ne vont pas passer facilement, du moins rapidement, d'une industrie à l'autre. Cela requiert des moyens financiers, structurels, humains, technologiques... trop considérables pour des entreprises de cette taille. 
La stabilité supposée des groupes de besoins satisfaits par les PME fait que cette variable ne sera pas reconnue comme facteur de discrimination de l'action de développement des PME.

Contrairement aux groupes de besoins, il est raisonnable de croire que les PME agissent assez souvent sur la zone géographique qu'elles desservent. Assez fréquemment, en tout cas, pour que cette variable participe à la discrimination des actions de développement des PME sur leurs activités produit/ marché.

Les travaux empiriques effectués auprès de PME vont dans ce sens. Par leurs actions sur la zone géographique qu'elles desservent, les PME peuvent se permettre une certaine croissance sans s'engager dans de nouvelles productions; en d'autres termes, sans avoir à supporter des investissements lourds, une politique de recherche, une politique de formation technologique. Elles peuvent se permettre au contraire de mettre à profit le savoir-faire technologique qu'elles ont acquis. En outre, les changements géographiques s'harmonisent avec une caractéristique qui fait la force des PME: la flexibilité.

Compte tenu de ces remarques sur les changements par rapport à l'étendue de la zone géographique, celle-ci sera considérée comme une variable discriminante des actions de développement des PME sur leurs activités produit/ marché.

En référence à l'orientation possible des changements, fixée préalablement, les actions de développement possibles des PME sur leurs activités produit/marché se définissent de la façon suivante:

repli ou maintien ou expansion :

- du nombre de produits ;

- de la diversité des produits ;

- du nombre de groupes de clients ;

- de l'étendue de la zone géographique.

L'intérêt de cette définition est qu'elle reconnaît aux PME la possibilité d'agir de façon indépendante sur trois des quatre critères de définition de la notion d'activité produit/marché qui ont été retenus (produits, groupes de clients et zone géographique), plutôt qu'une action globale qui semble peu plausible pour la plupart d'entre elles.

\subsubsection{Mesure des actions de développement}

Les actions de développement des PME seront mesurées en considérant, pour chacune des trois variables, les principales options de développement qui peuvent exister en tenant compte des sous-critères qui les caractérisent (nombre et diversité). 
Pour les produits, les actions qui ont été admises sont celles-ci :

- promotion des produits actuels ayant une forte contribution ;

- développement de nouvelles applications pour les produits existants ;

- modification des produits existants ;

- réduction du nombre de produits à l'intérieur des groupes de produits ;

- réduction du nombre de groupes de produits ;

- nouveaux produits à l'intérieur des mêmes groupes de produits ;

- nouveaux groupes de produits à l'intérieur de la même branche ;

- nouveaux groupes de produits pour de nouveaux types de branches;

- aucun changement de produits.

Les actions relatives aux groupes de clients, plus exactement par rapport au nombre, qui ont été choisies sont celles-ci :

- nouveaux groupes de clients ;

- abandon de groupes de clients ;

- aucun changement.

Les actions qui ont été envisagées concernant l'étendue géographique sont les suivantes :

- abandon ou réduction du marché national ;

- abandon ou réduction des marchés étrangers ;

- extension sur le marché national ;

- début des activités d'exportation ;

- entrée sur de nouveaux marchés étrangers ;

- extension des marchés étrangers ;

- aucun changement.

La mesure des actions dans lesquelles se sont lancées les PME étudiées se fera à partir de l'examen des réponses apportées par celles-ci aux différentes options de développement retenues.

L'information ainsi recueillie sera du type présence/absence.

Une entreprise peut, par exemple, avoir réduit le nombre de ses produits à l'intérieur des groupes de produits tout en développant de nouvelles applications pour des produits existants et n'avoir rien changé au niveau de ses groupes de clients et s'être, par contre, lancée dans l'exportation.

Une autre entreprise peut avoir créé de nouveaux produits à l'intérieur de la même branche pour de nouveaux groupes de clients, sans rien changer à l'étendue de sa zone géographique. 
Ces informations du type présence/absence seront les indicateurs des actions de développement suivies par les entreprises.

En plus de la mesure des types d'actions entreprises, il est intéressant de mesurer l'intensité de ces actions.

L'intensité des actions de développement menées par les PME sur leurs activités produit/marché sera définie dans l'étude comme le nombre d'actions dans lesquelles se sont engagées les entreprises rencontrées pour ce qui est des produits, des groupes de clients et de la zone géographique pris individuellement et globalement.

Soit les huit variables, V1 à V8, qui identifient les options de développement relatives aux produits, excepté l'alternative «aucun changement», les deux variables, V10 et V11, qui identifient les variables correspondant aux actions de développement possibles par rapport aux groupes de clients, excepté l'alternative «aucun changement » et enfin les six variables, V13 à V18, qui identifient les options de développement relatives à la zone géographique, excepté l'alternative «aucun changement», les indicateurs de l'intensité stratégique des actions de développement des PME concernant leurs activités produit/marché pourront alors s'écrire de la manière suivante:

$$
\mathrm{ISP}=\frac{\sum_{p=1}^{n} \mathrm{~V}_{p}}{8}
$$

où : $\quad V_{p}$ est l'action de développement par rapport aux produits, effectivement mise en œuvre

et : $\quad n$ le nombre d'actions de développement par rapport aux produits dans lesquelles l'entreprise s'est engagée.

$\mathrm{ISC}=\frac{\sum_{c=1}^{n} \mathrm{~V}_{c}}{2}$

où : $\quad V_{c}$ est l'action de développement par rapport aux groupes de clients, effectivement mise en œuvre

et : $\quad n$ le nombre d'actions de développement par rapport aux groupes de clients dans lesquelles l'entreprise s'est engagée.

$$
\mathrm{ISG}=\frac{\sum_{g=1}^{n} \mathrm{~V}_{g}}{6}
$$


où : $\quad V_{g}$ est l'action de développement par rapport à la zone géographique effectivement mise en œuvre

et : $\quad n$ le nombre d'actions de développement par rapport à la zone géographique dans lesquelles l'entreprise s'est engagée.

ISP, ISC et ISG ont une amplitude de [0 -1] qui les rend additifs et comparables. L'intensité globale des actions de développement peut dont s'écrire:

$$
\mathrm{IS}=\frac{\mathrm{ISP}+\mathrm{ISC}+\mathrm{ISG}}{3}
$$

L'amplitude est également [0 - 1].

L'intensité stratégique par rapport aux produits, aux groupes de clients, à la zone géographique et l'intensité globale seront d'autant plus fortes que la valeur d'ISP, d'ISC, d'ISG et d'IS sera élevée.

Pour déterminer cette intensité stratégique de l'entreprise, en d'autres termes, son dynamisme par rapport aux produits, aux clients et à la zone géographique, l'échelle retenue précédemment pour évaluer le niveau de diversification sera choisie.

L'intérêt d'une mesure de la dynamique stratégique décomposée et non seulement globale permet de montrer, par exemple, qu'une entreprise peut être dynamique sur le plan de ses produits et statique, voire en repli sur le plan de ses groupes de clients et sa zone géographique, et inversement.

La nouvelle mesure de la stratégie de diversification des PME étant proposée, son application concrète est possible. Pour servir de support à cette application, 22 PME du secteur laitier ont été étudiées.

\section{Cinq types de comportement de diversification}

À partir des informations recueillies auprès de 22 PME du secteur laitier de l'Ouest de la France, la mesure de la diversification des PME et de son développement a pu être appliquée.

Les informations ont été obtenues à l'aide d'interviews réalisées sous forme de questionnaires auprès des dirigeants des entreprises. La collecte des informations s'est faite en deux étapes. La première, réalisée en 1985, fut consacrée à la collecte d'informations relatives à un grand nombre de variables avec un questionnaire élaboré dans le cadre du projet STRATOS (Bamberger, 1983): identité de l'entreprise et du dirigeant, ses valeurs et attitudes, ses objectifs et 
ceux de l'entreprise, caractéristiques de l'environnement, stratégies de l'entreprise et performance de l'entreprise. Elle permettait une première approche du terrain.

La seconde étape, menée en 1986, fut consacrée à la recherche d'informations précises concernant la nature de la stratégie de diversification à l'aide d'un questionnaire spécialement élaboré pour l'étude.

À partir des neuf variables, DI, DS, DV, DP, DC, DB, DG, DM et D, la nature du comportement de diversification des $22 \mathrm{PME}$ a pu être déterminée. La classification ascendante hiérarchique, méthode d'analyse typologique, a été retenue pour dégager la nature du comportement de diversification des PME rencontrées. La méthode de WARD est la mesure de proximité employée par le logiciel SPAD utilisé (Lebart et Morineau, 1985).

Les actions de développement menées par les entreprises, compte tenu de la nature de leur comportement de diversification, ont été conjointement analysées à partir des variables V1 à V18, indicateurs des changements effectués, et à partir d'ISP, ISC, ISG et IS, indicateurs de l'intensité des changements effectués, soit par le T-test soit par l'analyse de la variance.

De ces analyses, cinq types de comportement de diversification ont été dégagés :

- type 1 (6 entreprises) spécialisation produits et diversification commerciale peu dynamique ;

- type 2 (7 entreprises) spécialisation produits et diversification commerciale d'exportation peu dynamique ;

- type 3 (3 entreprises) diversification produits et diversification commerciale d'exportation dynamiques ;

- type 4 (4 entreprises) diversification produits et diversification commerciale dynamiques ;

- type 5 ( 2 entreprises) diversification produits et diversification commerciale nationale dynamiques.

\subsection{Type 1 : Spécialisation produits et diversification commerciale peu dynamique}

Ces entreprises ont un faible niveau de diversification globale et sont modérées dans leur développement. Leur diversification est souvent orientée vers les clients nationaux avec une certaine diversification internationale.

Leur diversification produits se limite au secteur laitier et les produits sont relativement liés, leurs compétences technologiques sont donc étroites.En outre, 
leurs actions de développement se caractérisent par le maintien de leurs groupes de produits et de leurs clients nationaux parallèlement à une expansion de leurs produits et de leur zone géographique nationale, voire internationale.

C'est le cas de cette entreprise qui, jusqu'en 1983, fabriquait du beurre, de la crème et du fromage à pâte molle et qui s'est lancée dans le fromage frais par lequel elle cherche à étendre son implantion nationale. Ce nouveau produit, qui requiert peu de compétences technologiques nouvelles, offre à l'entreprise l'avantage d'être distribué par le même réseau que ses autres produits et d'être en phase d'évolution et de bonne rentabilité, comparé à ses autres produits.

\subsection{Type 2 : Spécialisation produits et diversification commerciale d'exportation peu dynamique}

Ces entreprises ont un faible niveau de diversification globale et sont modérées dans leur développement. Leur diversification, bien qu'équitablement tournée vers leurs produits, clients et zone géographique internationale, est assez fortement orientée vers l'internationalisation. Leurs actions de développement se caractérisent par le maintien de leurs groupes de produits parallèlement à une expansion de leurs groupes de clients et de leur zone géographique nationale et internationale.

C'est le cas d'une entreprise qui fabrique du lait UHT, du fromage à pâte molle et à pâte pressée non cuite, qu'elle vend à des distributeurs, des grossistes et des détaillants, ainsi qu'à des entreprises industrielles, et dont elle exporte environ $40 \%$ de sa production.

\subsection{Type 3 : Diversification produits et diversification d'exportation dynamiques}

Ces entreprises ont une diversification globale d'un niveau moyen et elles sont relativement dynamiques. Leur diversification est tournée vers les clients nationaux et internationaux avec des groupes de produits relevant de compétences technologiques étroites. Leurs actions de développement se caractérisent par le maintien de leurs groupes de clients et l'expansion de leurs groupes de produits, a fortiori vers de nouvelles branches, et de leur zone géographique nationale et internationale.

C'est le cas d'une entreprise qui réalise $80 \%$ de son chiffre d'affaires au sein du secteur laitier avec des produits d'une technologie simple: lait, poudre de beurre, crème et qui, pour chacun d'eux, a une activité d'exportation. Elle a aussi lancé son activité jus de fruit durant ces dernières années et elle a développé ses exportations. 
Il est à noter que ces trois entreprises, qui ont quasiment toutes leur activité dans le secteur laitier, ont pour seule activité en dehors du lait, le jus de fruit. Ceci s'explique par le fait que cette production repose sur le même principe technologique que le lait UHT qu'elles fabriquent toutes. Leur faible diversification intersecteurs se fonde donc sur une compétence technologique qu'elles maîtrisent pour le lait.

\subsection{Type 4 : Diversification produits et diversification commerciale dynamiques}

Ces entreprises ont une diversification globale d'un niveau moyen et elles sont dynamiques. Leur diversification est surtout tournée vers les clients nationaux et internationaux et les groupes de produits, a fortiori en dehors du secteur laitier, donc avec des compétences technologiques relativement larges. Leurs actions de développement se caractérisent par le maintien de leurs groupes de produits et l'expansion ou le repli de leurs groupes de clients et de leur zone géographique nationale ou internationale.

C'est le cas d'une entreprise qui, en plus de son activité laitière, a une activité viande, céréales et approvisionnement en engrais. Elle fonde sa forte implantation en dehors du secteur laitier sur la même logistique d'approvisionnement, puisque ses fournisseurs sont toujours les agriculteurs. Elle connaît les problèmes de contrôle de qualité et de quantité de production dans tous les cas. Sa diversification intersecteurs repose donc sur des technologies d'approvisionnement identiques. Ses technologies de transformation sont, par contre, différentes et ses types de clients sont, quant à eux, identiques.

\subsection{Type 5 : Diversification produits et diversification commerciale dynamiques}

Ces entreprises ont une diversification globale d'un niveau moyen et elles sont dynamiques. Leur diversification est surtout tournée vers les groupes de clients nationaux et les groupes de produits avec des compétences technologiques relativement larges. Leurs actions de développement se caractérisent par l'expansion de leurs groupes de produits, a fortiori dans de nouvelles branches, de leurs groupes de clients et de leur zone géographique nationale et internationale.

C'est le cas d'une des deux entreprises qui, jusqu'en 1984, produisait différents fromages, des laits fermentés, du fromage frais et qui, en 1985, a lancé une ligne de produits à base de soja. Cette nouvelle production requérait à la fois des compétences technologiques nouvelles et une dynamique commerciale pour 
toucher une large clientèle. Elle a impliqué des changements profonds, notamment, pour les matières premières et donc, pour la logistique de l'approvisionnement. Bien que cette entreprise cherche à développer son implantation internationale, elle met surtout l'accent sur un développement de clientèle et des technologies sur le plan national.

Il ressort de cette analyse que les PME observées ont un niveau global de diversification soit faible, soit moyen. Ce niveau de diversification a pu être observé grâce à une approche dimensionnelle des activités produit/marché des PME et non globale, sans quoi la diversification des PME étudiées se traduirait souvent par une spécialisation.

De même, elle montre que les PME peuvent agir sur les trois dimensions de leurs activités ou seulement sur une ou deux comme les entreprises des types 2 et 3 , d'où l'intérêt d'étudier le comportement de diversification et les actions de développement par dimension et non globalement.

Outre ces premiers éléments, cette étude souligne l'évolution du comportement stratégique des PME. Ainsi, les entreprises des types 1 et 2, peu diversifiées et peu dynamiques, privilégient le marché national et international au détriment des produits, en d'autres termes, des compétences technologiques, contrairement aux entreprises des types 3,4 et 5 .

Cette typologie va dans le sens des résultats de l'étude de Mussault et Perquier (1979) selon lesquels, les PME se développent soit par une spécialisation de leurs produits, accompagnée d'une extension géographique, a fortiori internationale, soit par un élargissement de leurs produits basé sur des compétences technologiques. Elle rejoint également les réflexions de Martinet (1985), appuyées par les résultats de l'étude du CEPME (1988), lorsqu'il oppose l'internationalisation à la diversification nationale.

La typologie met effectivement en évidence l'idée que les PME se développent selon ces deux orientations, les produits et les marchés. Toutefois, il apparaît dans la typologie dégagée que l'alternative entre ces deux orientations est le reflet d'une phase d'évolution dans le niveau de diversification.

Les PME rencontrées s'orientent d'abord vers les marchés puis vers les produits; en d'autres termes, elles développent dans un deuxième temps leurs compétences technologiques. Les cinq types de comportement de diversification relevés correspondent donc à un chemin d'expansion des PME.

Cette typologie obtenue à partir de la proposition d'une mesure de la diversification des PME, spécifique aux caractéristiques de ces entreprises, atteste la nécessité de mettre en place des outils d'analyse qui leur soient adaptés. Sans quoi, leur comportement stratégique paraîtra toujours quasi homogène et toute analyse structurelle d'un secteur sera biaisée. 


\section{Conclusion}

L'objet de cette recherche était de proposer un outil d'analyse stratégique selon une approche sectorielle adapté aux caractéristiques des PME. En ce sens, une définition et une mesure de la nature de la diversification des PME et de son développement ont été élaborées et appliquées auprès de 22 PME du secteur laitier.

Sur le plan méthodologique, il importe de retenir de ces travaux qu'il faut:

- connaître le secteur étudié, notamment, sous l'angle technologique, même si cela implique un investissement de longue durée, pour définir correctement l'activité du secteur concerné ;

- définir la notion d'activité des PME avant toute démarche visant à définir et à mesurer la diversification ;

- combiner l'approche statique et l'approche dynamique de la diversification pour avoir une idée la plus proche possible de la réalité stratégique des PME ;

- avoir une approche dimensionnelle de la diversification et non globale pour éviter que l'étude n'aboutisse au constat que les PME sont toutes spécialisées ;

- envisager que les PME peuvent non seulement maintenir et élargir leur activité, mais aussi la recentrer pour la consolider sans pour autant signifier qu'elles sont en difficulté.

Sur le plan des résultats, il ressort essentiellement que les 22 PME rencontrées orientent leur diversification selon leur phase d'évolution: d'abord vers les marchés nationaux, puis internationaux et ensuite, vers les compétences technologiques, fondement de la diversification produits.

Deux questions émergent au terme de ce travail qu'il faudrait aborder dans une prochaine étude:

- Peut-on associer la diversification à dominante marché à l'activité « vente d'heures » et la diversification à dominante produit à l'activité " vente de produit propre », voire de « spécialité » pour reprendre l'analyse de Brechet (1990)?

- Existe-t-il un lien entre le type de diversification ou la phase d'expansion et la performance de l'entreprise? 


\section{Bibliographie}

ABELL, D.K. (1980), Defining the Business : The Starting Point of Strategic Planning, Englewood Cliffs, N. J., Prentice-Hall.

ANSOFF, H.L. (1965), Corporate Strategy, New York, McGraw-Hill. Traduction française, Stratégie du développement de l'entreprise, Paris, Hommes et Techniques, 1968.

BAMBERger, I. et M. MERDJI (1980), «Situations et comportements stratégiques des petites et moyennes entreprises », Cahiers Stratégie et Organisation, $\mathrm{n}^{\circ} 4$, Rennes.

BAMBERger, I. (1983), « Les valeurs des dirigeants de PME et leur influence sur le comportement stratégique et la performance de leurs entreprises », Sciences de Gestion, p. 1353-1383.

BRECHET, J.P. (1990), « Pour une analyse stratégique adaptée à la P.M.I. », Revue Française de Gestion, juin-juil.-août, p. 19-29.

CHICHA, J. et P.A. JULIEN (1979), "Les stratégies des PME et leur adaptation au changement », GREPME, Université du Québec à Trois-Rivières.

CRÉDIT D'ÉQUIPEMENT DES PME (CEPME) (1981), Les petites et moyennes industries en 1981, Paris.

CRÉDIT D'ÉQUIPEMENT DES PME, (1988), Les petites et moyennes entreprises, investissement, stratégies, financement, Paris.

HuNT, M.S. (1972), Competition in the Major Home Appliance Industry 1960-1970, Thèse de doctorat non publiée, Université Harvard.

LEBART, L. et A. MORINEAU (1985), SPAD, Système portable pour l'analyse des données, CESIA.

LOUITRI, A. (1984), Stratégies de diversification et structures organisationnelles des groupes industriels. Profils, facteurs et performances (1970-1980), Thèse d'État, Bordeaux I.

MARTINET, A.Ch. (1985), "Internationalisation ou diversification domestique de la PME : instruction d'un dilemme stratégique », Les Cahiers Lyonnais de Recherche en Gestion, $\mathrm{n}^{\circ}$ 7, juin, p. 124-141.

MusSAult, O. et D. PERQuiER (1979), « Cinq stratégies d'entreprise moyenne », Revue Française de Gestion, sept.-oct., p. 74-80.

ORSONI, J. (1985), «Les PME face aux nouvelles conditions de l'échange international », Cahiers de Recherche de l'I.A.E. de Lille, $\mathrm{n}^{\circ}$ 85/8.

PENROSE, E.T. (1963), Facteurs, conditions et mécanismes de la croissance des entreprises, Paris, Hommes et Techniques, p. 101. 
PORTER, M.E. (1980), Choix stratégiques et concurrence, Paris, Économica.

RAMANUJAN, V. et P. VARADARAJAN (1989), « Research on Corporate Diversification : A Synthesis », Strategic Management Journal, vol. 10, $\mathrm{n}^{\circ}$ 6, nov.-déc., p. $523-551$.

RoвIC, P. (1989), Stratégie de diversification des PME, mesure et analyse; le cas de $P M E$ du secteur laitier, Thèse d'Université, Rennes I.

RotHSCHILD, W.E. (1979), Strategic Alternatives, New York, McGraw-Hill.

Rumelt, R.P. (1974), Strategy, Structure and Economic Performance, Cambridge, Mass., Harvard University Press.

SAlter, M.S. et W.A. WeINHOLD (1979), Diversification Through Acquisition, New York, The Free Press. 